Published in final edited form as:

ACS Nano. 2019 December 24; 13(12): 14024-14032. doi:10.1021/acsnano.9b06326.

\title{
In Vivo Photoacoustic Lifetime Based Oxygen Imaging with Tumor Targeted G2 Polyacrylamide Nanosonophores
}

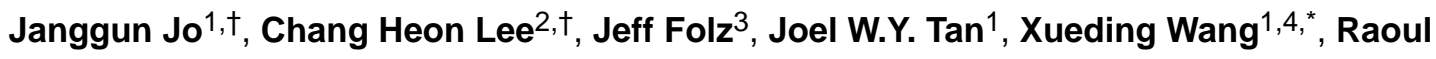 \\ Kopelman ${ }^{1,2,3, *}$ \\ ${ }^{1}$ Department of Biomedical Engineering, University of Michigan, Ann Arbor, Michigan, 48109, \\ USA. \\ 2Department of Chemistry, University of Michigan, Ann Arbor, Michigan, 48109, USA. \\ ${ }^{3}$ Biophysics Program, University of Michigan, Ann Arbor, Michigan, 48109, USA. \\ ${ }^{4}$ Department of Radiology, University of Michigan Medical School, Ann Arbor, Michigan, 48109 \\ USA.
}

\begin{abstract}
Lifetime imaging methods using phosphorescence quenching by oxygen for molecular oxygen concentration measurement have been developed and used for non-invasive oxygen monitoring. This study reports photoacoustic (PA) oxygen imaging powered by polyacrylamide (PAAm) hydrogel nanoparticle (NP) which offer advantages including improved biocompatibility, reduced toxicity, and active tumor targeting. A known oxygen indicator, oxyphor G2, was conjugated with the matrix of the NPs, giving G2-PAA NPs, followed by PEGylation for biocompatibility and F3 surface modification for tumor targeting. Using two lasers providing pump and probe pulses, respectively, PA imaging was performed so as to quantitatively map the oxygen concentration in biological tissues in vivo, including cancer tumors and normal thigh muscles. Furthermore, via the imaging at the pump wavelength and two additional wavelengths, the accumulation of the G2-PAA NPs in the tumors were also determined. The successful imaging experiment accomplished on animal models renders a method for in vivo non-invasive imaging and assessment of hypoxic tumor microenvironments, which is critical for assessing cancer progression, metastasis, and treatment.
\end{abstract}

\section{Graphical Abstract}

\footnotetext{
*Corresponding Authors Drs. Wang and Kopelman are corresponding authors. Xueding Wang, PhD - xdwang @umich.edu. Telephone: 734-647-2728.; Raoul Kopelman, PhD - kopelman@ umich.edu. Telephone: 734-764-7541.

† These authors contributed equally to this work.

Present Addresses

University of Michigan, 500 S State St, Ann Arbor, MI 48109, USA

Author Contributions

The manuscript was written through contributions from all authors. All authors have given approval to the final version of the manuscript. The authors, Drs. Jo and Lee, contributed equally.

Supporting Information

Figure S1:G2-PAA NP characterization with Hydrodynamic size; Figure S2: TEM image of G2-PAA NPs; Figure S3: Cell viability; Figure S4: the ${ }^{1} \mathrm{H}$ NMR spectrum; Table S1: measured zeta-potentials for blank PAAm NP (PDF)

Financial interest statements

The authors declare no competing financial interest.
} 


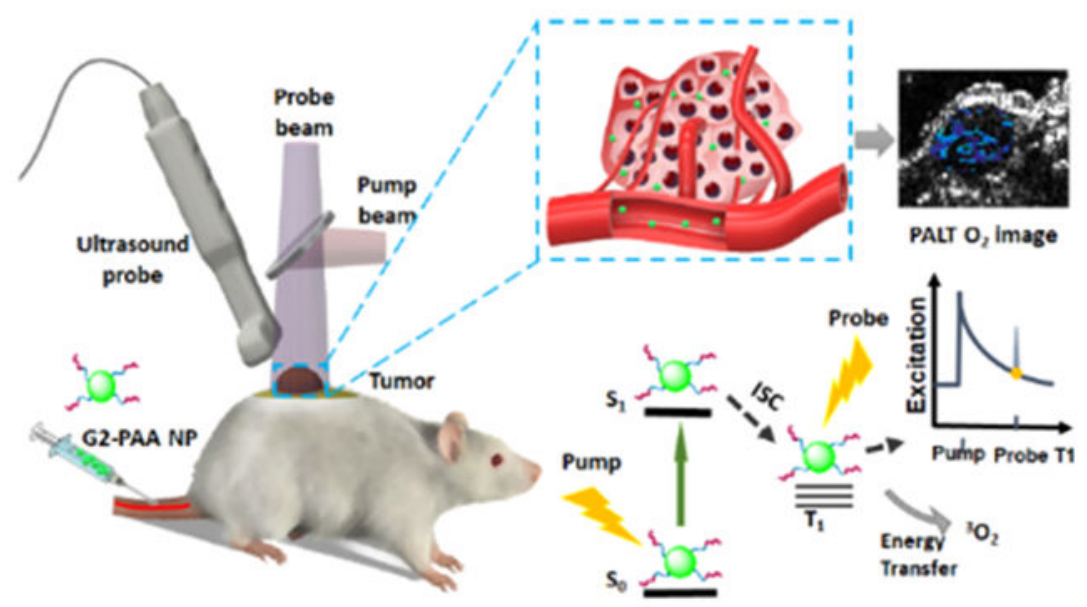

\section{Keywords}

photoacoustic; lifetime; oxygen; nanoparticle; oxyphor; tumor imaging

Oxygen is a highly reactive element capable of combining with most other elements, and is essential for most aerobic organisms. An abnormal oxygen concentration is one of the most prevalent factors for various pathologies, including cancer. Hypoxia (low oxygen concentration) is a hallmark characteristic for the tumor microenvironment, and its association with cancer pathologies, such as angiogenesis and metastasis, has been targeted for therapies. ${ }^{1-4}$ Despite increasing attention to the hypoxic tumor microenvironment, there is lack of non-invasive tools for quantitative oxygen mapping in an entire solid tumor in vivo. Notably, no biopsy can provide such information. Oxygen imaging of tumors in vivo has been attempted through magnetic resonance imaging (MRI), positron emission tomography (PET), and optical imaging. ${ }^{5-7}$ MRI and PET, however, are expensive techniques, and of limited access. Conventional optical imaging, such as fluorescence microscopy, offers a low-cost solution for oxygen mapping in vivo and in vitro. Although a variety of optical oxygen sensors have been developed and many applications have been explored, conventional optical imaging, in general, suffers from the overwhelming optical scattering inside biological tissues. As a result, high-resolution microscopic imaging modalities are limited to either cellular studies in vitro or very superficial tissues in vivo. Other optical imaging modalities working in the diffusion region, although being capable of covering an entire solid tumor, cannot offer sufficient spatial resolution required for studying heterogeneous tumor microenvironment. To overcome these limitations, the emerging photoacoustic (PA) imaging technique has been recently developed for chemical imaging of tumor microenvironmental parameters such as tissue oxygen concentration. ${ }^{8-18}$

In PA imaging, a pulsed light source excites targeted chromophores inside the body, which can be either internal chromophores, such as hemoglobin, or externally injected agents, such as dyes. The absorbed light energy is turned into local thermal expansion, ultimately forming ultrasound (US) waves which can be detected by highly sensitive US transducers. Since US waves exhibit significantly less scattering and diffraction in biological tissues, in comparison to light, the image quality of PA imaging is determined by the detection of the 
laser-induced ultrasound waves, instead of by the diffusing photons. PA imaging, based on the endogenous optical contrast, allows quantitative imaging of blood oxygenation by utilizing the optical absorption differences between the oxygenated hemoglobin and the deoxygenated hemoglobin. ${ }^{19-21}$ However, it is important to note that the blood oxygenation level provides only an indirect measure of the tissue oxygenation level.

Aiming at assessing tissue oxygenation levels directly, photoacoustic lifetime (PALT) based measurement was proposed which utilizes phosphorescence based oxygen indicators. ${ }^{11-15}$ Phosphorescence exhibits longer emission times (micro-second to milli-second), in comparison to typical fluorescence emission times on the order of nano-seconds, due to the energy decay process occurring through a forbidden "intersystem crossing" transition, from its first excited singlet state $\left(S_{1}\right)$ to the first excited triplet state $\left(T_{0}\right)$ (Figure $\left.1 b\right)$. The energy of the first excited triplet state can be directly transferred into oxygen molecules, which exhibit a triplet ground state. As the energy during phosphorescence emission is transferred into the nearby oxygen molecules, the lifetime of the phosphorescence emission is shortened with higher ambient oxygen partial pressure, in an oxygen concentration dependent manner. This phenomenon has been well established for fluorescence based oxygen concentration measurements. ${ }^{5}$ However, PALT based on the same phenomenon has been studied only recently. $7,11-15$

PALT utilizes two laser pulses, respectively from the pump and the probe beams (Figure 1a). The pump pulse excites the sensing dye, which quickly decays into its first excited triplet state, through intersystem crossing. Then the probe pulse excites the first triplet state of the sensing dye, which is similar to transient absorption spectroscopy. The PA signal from the triplet state absorption is later detected by an US transducer (Figure 1). By varying the delay time between the pump and the probe pulses, the exponential decay of the phosphorescence can be probed. This decay rate is known to linearly correlate with the ambient oxygen concentration, resulting from a first order reaction (Figures $1 \mathrm{~b}$ and $1 \mathrm{c}$ ). PALT has been mostly applied using free dyes. Thus, most of the previous studies have been conducted by direct injection of the dye into the designated image regions. ${ }^{11-15}$

Here, we further extended the PALT technique to make it more suitable for in vivo oxygen imaging by using the commercially available phosphorescence indicator, oxyphor G2, after its conjugation to polyacrylamide nanoparticles (PAAm NPs). The oxyphor G2 dye has a much longer phosphorescence lifetime (i.e. 254-281 $\mu$ s) ${ }^{22}$ compared to other dyes, for example methylene blue (i.e. $79.5 \mu \mathrm{s}),{ }^{23-25}$ which is beneficial for PALT based imaging. The PEGylated nanoparticle matrix protects the G2 sensing dye from non-specific interaction with other proteins, such as albumin, or other unwanted biochemical interactions. These biochemical interactions may cause changes in the optical properties of the sensing dyes. ${ }^{26}$ In addition, the surface of the nanoparticle is also conjugated with the tumor homing F3peptide, thus allowing selective targeting toward tumors by intravenous injection. ${ }^{15}$ The oxygen sensing capability of the G2-PAA NP was confirmed in vitro. Then, via a study on a mouse model in vivo, this capability of PALT in imaging tumor targeted tissue oxygen levels was demonstrated. 


\section{RESULTS/DISCUSSION}

\section{$\mathrm{O}_{2}$ sensing nanoparticle (G2-PAA NP) and PALT capability of the G2-PAA NP}

Oxyphor $\mathrm{G} 2$, a phosphorescence based $\mathrm{O}_{2}$ sensitive dye, has excitation and phosphorescence emission wavelengths at $632 \mathrm{~nm}$ and $800 \mathrm{~nm}$, respectively, which are within the optimal optical window of biological tissues. ${ }^{22}$ In a previous study, oxyphor G2 has shown its potential as a PALT indicator. ${ }^{15}$ The oxyphor G2 contained 16 carboxylic acid groups on the surface, allowing surface modification and conjugation to the nanoparticle matrix for more stable in vivo $\mathrm{O}_{2}$ monitoring. ${ }^{27,28}$ The oxyphor $\mathrm{G} 2$ was preconjugated to one of the monomers prior to nanoparticle formation so as to minimize leaching of the dye during the measurements or imaging process in vivo (Figure 2a). The surface of the nanoparticles was PEGylated to maximize nanoparticle circulation time and further activated with the tumor homing F3-peptides for achieving targeted delivery of the nanosensors in vivo after intravenous injection.

Oxyphor G2, solely on its own, is a small molecule, and cannot avoid interactions with other bio-macromolecules such as albumin, the most abundant protein in the body. Here, we tested the G2 free dye and the G2-PAA NPs mixing with human serum albumin (HSA) at $4 \mathrm{mg} / \mathrm{ml}$, the relevant physiological concentration (Figure 2b). Fluorescence emission of the oxyphor G2 is known to be enhanced by several folds upon addition of the albumin. ${ }^{22}$ When a fluorophore absorbs photon energy from an excitation beam, the dye releases its absorbed energy through both fluorescence and non-radiative decay. The later one can be detected photoacoustically. Thus, the change in fluorescence emission can influence PA signals collected during in vivo PALT experiments. On the other hand, the fluorescence emission of the G2-PAA NPs is not changed obviously in the presence of the albumin, as shown in Figure $2 \mathrm{~b} .{ }^{26}$ The polyacrylamide matrix entraps the oxyphor $\mathrm{G} 2$ and protects it from direct interaction with other macromolecules, thus protecting the dye from altering its optical properties in the in vivo environments. At the same time, other potential interactions of the dye with the in vivo biological environments are also avoided.

The two excitation wavelengths for the pump pulse and the probe pulse were experimentally determined. Figure $2 \mathrm{c}$ shows the PA lifetime signal amplitude as a function of the wavelengths of the pump and the probe pulses. The delay time between the pump and the probe pulses was set at $10 \mu$ s. The maximal PA lifetime signal amplitude was observed with a 630-nm pump excitation and a 920-nm probe excitation. Thus, we used these two wavelengths in later PALT studies.

\section{Oxygen calibration using G2-PAA NPs at different concentrations}

The excited triplet state of the oxyphor G2 decays exponentially, while the rate of decay is linearly correlated with the ambient oxygen concentration. ${ }^{13-15,29}$ PALT is able to determine the triplet state lifetime of the oxyphor G2 and the decay rate by varying the delay time between the pump and the probe pulses. In Figure 3, PA signals correspondent to the triplet state absorption of the G2-PAA NPs with different delays (10, 20, 30, 40, and $50 \mu$ s, respectively) between the pump and the probe pulses are shown. Each panel shows the result for different oxygen concentrations from $2 \%$ to $20 \%$, grouped into four ranges. To examine 
whether the PALT is dependent on the concentration of the G2-PAA NPs, this measurement was conducted for G2-PAA NPs at different concentrations $(2,5$, and $20 \mathrm{mg} / \mathrm{ml}$, respectively), as well as for $5 \mathrm{mg} / \mathrm{ml} \mathrm{G2-PAA} \mathrm{NPs} \mathrm{mixed} \mathrm{with} \mathrm{HSA}(4 \mathrm{mg} / \mathrm{ml})$. Exponential decay fittings were performed for each oxygen concentration and each G2-PAA NP concentration to extract the decay rates, as shown by the solid curve in each panel in Figure 3. As we can notice, the decay curves are largely different when the ambient oxygen concentrations are at different levels, while the decay curves stay consistent for different G2PAA NP concentrations as well as with the presence of HSA.

As we planned to intravenously inject G2-PAA NP into tumor xenograft mice, we confirmed that our measurement of oxygen concentration is independent of G2-PAA NP concentration. The quantified decay rates as a function of the ambient oxygen concentration are shown in Figure 4, with a - d showing the results for different G2-PAA NP concentrations as well as with the presence of HSA. In each panel, a linear fit can be made, demonstrating that the decay rate is linearly correlated with the ambient oxygen concentration. The fitting lines in the four panels in a - $d$ are compared in Figure 4e. We can see that, for different G2-PAA NP concentrations and with the presence of HSA, the decay rates are similar. All the PA measurement points in Figure 4a - 4d are included in Figure 4f. A linear fit is made, without considering the G2-PAA NP concentration and the presence of HSA. This linear fit in Figure $4 \mathrm{f}$ was used as a calibration for later quantitative imaging of oxygen concentrations in biological samples in vivo. This study in vitro demonstrated that the PALT based measurement of oxygen concentration is independent of the G2-PAA NP concentration, and is not affected by the presence of HSA.

\section{In vivo PALT images of control muscles}

Prior to tumor imaging, PALT images of control muscles in vivo were taken. The G2-PAA NPs were subcutaneously injected locally into the thigh muscle with five shots surrounding the imaging area. Hypoxia in the muscle was induced by lowering the oxygen content in the breathing gas from $100 \%$ to $10 \%$ (90\% nitrogen and $10 \%$ oxygen). Figure 5 shows the PALT based oxygen images in the thigh muscle of an animal, where (a)-(c) are for the results correspondent to $10 \%, 20 \%$, and $100 \%$ oxygen contents in the breathing gas. The pseudocolor PALT images demonstrating the oxygen concentrations in the imaged muscle area are superimposed on the gray-scale US image. Any pixel with quantified oxygen concentration beyond the range of $0-10 \%$ was considered as error, where no color was assigned. This experiment was repeated on four animals $(n=4)$. For each animal, the oxygen concentration in the imaged muscle area was first averaged. Then, with the averaged measurements from the four animals, the average and the standard deviation were calculated for each situation. As shown in Figure 5d, the averaged oxygen concentration levels in the thigh muscles of the 4 animals were $1.49 \pm 1.48(\%), 3.51 \pm 1.64(\%)$, and $5.65 \pm 0.60(\%)$, respectively, for $10 \%$, $20 \%$, and $100 \%$ oxygen contents in the breathing gas. As expected, the tissue oxygen levels decreased significantly as the oxygen content in the inhaling gas decreased.

\section{In vivo PALT images of tumors}

Figure $6 \mathrm{a}-6 \mathrm{c}$ show the example imaging results from a mouse tumor. In the US image in Figure $6 \mathrm{a}$, the tumor region can be recognized clearly. Figure $6 \mathrm{~b}$ shows the distribution of 
the G2-PAA NPs in the imaging area, which was derived from the three PA images at the three wavelengths $(576 \mathrm{~nm}, 584 \mathrm{~nm}$, and $630 \mathrm{~nm}$ ) by conducting multispectral PA imaging. This image confirmed the preferred accumulation of the G2-PAA NPs in the tumor area. The accumulation of G2-PAA NPs toward the tumor was also confirmed by the ex vivo fluorescence imaging of the tumor tissue and important organs harvested after the in vivo imaging experiments. As shown in Figure 7, the fluorescence images demonstrate that the G2-PAA NPs accumulated mostly in the tumor and the liver, which is consistent with our previous findings. ${ }^{26}$

The PALT image presenting the oxygen concentration in the tumor in vivo is shown in Figure $6 \mathrm{c}$. The oxygen image was obtained via the same image process using in PALT imaging of thigh muscle in vivo. Any pixel with quantified oxygen concentration beyond the range of $0-10 \%$ was considered as error, where no color was assigned. Additionally, any pixel with G2-PAA NP concentration under $0.01 \%$ was also excluded in the oxygen image. The functional imaging of tumor was repeated on four animals $(n=4)$. For each animal, the oxygen concentration in the imaged tumor area was first averaged. Then, with the averaged measurements from the four animals, the average oxygen saturation and the standard deviation in the tumors were calculated. Figure $6 \mathrm{~d}$ shows the oxygen concentrations in the tumors in comparison with the results in control muscles, both measured with $100 \%$ oxygen content in the breathing gas. As we can see, the oxygen concentrations in the tumors are significantly lower, with an average of $1.94 \pm 2.05(\%)$, compared to those in the thigh muscles with an average of $5.65 \pm 0.60(\%)$. To examine whether there is statistically significant difference between the tumors and the muscles, a two tailed t-test was performed using the built-in functions of the MATLAB (R2016b, Mathworks, Natick, Massachusetts), which achieved a p value $<0.01$.

\section{CONCLUSIONS}

A known PALT indicator, oxyphor G2, was conjugated and encapsulated with PAAm NPs, and the surface of the PAAm NPs were PEGylated and modified with tumor homing F3 peptides to grant active tumor targeting. Oxyphor G2, by itself, has been demonstrated to perform single point PALT measurements (not imaging) in vivo. ${ }^{15}$ However, as shown in Figure 2b, oxyphor G2 interacts with other bio-macromolecules, such as serum albumin, which alters its optical properties. Such changes can significantly complicate or void the functional measurements based on the detection of optical absorption or fluorescent spectrum. By conjugating and encapsulating the oxyphor G2 in the PAAm NPs, the optical properties of the oxyphor G2 stay consistent even in the presence of albumins, making quantitative oxygen measurement in vivo possible. This indicates that the nanoparticle matrix protects the oxyphor $\mathrm{G} 2$ dyes from direct interaction with the albumins. ${ }^{8}$ This technical advancement over the previous reported method based on the use of oxyphor G2 dye in its free style is crucial for future preclinical and clinical applications.

PEGylation has been widely studied over the past few decades and shown to maximize biocompatibility. ${ }^{30}$ In addition to simple PEGylation, we have previously studied the F3peptide conjugation (active targeting moiety) and found that it can increase the half-life of the PAAm NPs, i.e. surviving removal by the immune system, by around 4 folds in $9 \mathrm{~L}$ 
glioma tumor tissue in vivo (39 min vs. $123 \mathrm{~min}$ ). ${ }^{31}$ The nanoparticle matrix prevents undesirable interactions between the Oxyphor $\mathrm{G} 2$ and other bio-macromolecules (i.e. serum albumin) which can cause alteration of dye's optical and sensory properties. Powered by the G2-PAA NPs, PALT based oxygen concentration imaging can be achieved in biological samples in vivo. As demonstrated by experiments on mouse models, the change in oxygen concentration in the thigh muscle induced by adjusting the breathing gas was successfully imaged. The spatially distributed oxygen concentrations in solid tumors were mapped, and the significantly lower oxygen concentrations in the tumors in comparison to normal tissues were also validated. The depth of penetration of PA imaging depends on the excitation wavelengths. Our previous studies revealed that when we are using a $565 \mathrm{~nm}$ to $600 \mathrm{~nm}$ excitation source, accurate PA chemical imaging penetrates up to $6 \mathrm{~mm}$ in depth. ${ }^{26}$ We have previously reported PALT based measurements and imaging using methylene blue embedded polyacrylamide nanoparticles (MB-PAA NPs) and demonstrated an imaging depth of at least $5 \mathrm{~mm}$ in an in vivo mouse tumor model. ${ }^{23}$ As the pump and probe excitation wavelengths used for G2-PAA NP (630 nm pump and $920 \mathrm{~nm}$ probe) are similar to MB-PAA NP (660 nm pump and $840 \mathrm{~nm}$ probe), we expect a similar depth of at least 5 $\mathrm{mm}$ for PALT based oxygen imaging.

PA technique has been widely applied for in vivo non-invasive imaging of blood oxygenation. ${ }^{32,33}$ Based on the endogenous optical absorption contrast between oxygenated and deoxygenated hemoglobin, PA imaging working with multiple laser wavelengths is capable of detecting the percentage of oxygenated hemoglobin in total hemoglobin. This blood oxygenation level, however, only provides an indirect assessment of tissue oxygenation which is more important in evaluating tissue metabolic activities. It is also important to note that hypoxia and hypoxemia do not always occur together. ${ }^{34}$ PALT based oxygen imaging, however, provides a direct assessment of tissue oxygenation level, and offers a tool for evaluating hypoxia in cancerous tumor tissue. In addition, the tissue oxygen imaging method reported here can work for any locations where the nanosensors can be delivered, even in the necrotic core of a tumor where not much blood is present. The polyacrylamide based nanosensor not only increases biocompatibility of the oxygen sensing dye and prevents undesired interactions of the dye molecules with macromolecules, but also provides tumor targeting capability by modifying the surface with targeting moieties. In this work, the capability of this imaging technique was demonstrated via the study on a mouse tumor model. However, we believe that the same technique can be extended to many other diseases associated with hypoxia. To target other tissues or organs, exchanging the targeting moiety from $\mathrm{F} 3$ peptide to others is a relatively trivial process.

\section{MATERIALS/EXPERIMENTAL}

\section{Chemicals}

All chemicals were purchased from Sigma Aldrich or ThermoFisher Scientific unless otherwise noted. 


\section{Preparation of G2-PAA NPs}

The Oxyphor G2 (4 mg, Oxygen Enterprises, Ltd., Philadelphia, PA, USA.) was conjugated to 3-(aminopropyl) methacrylamide hydrochloride salt (APMA, $53.6 \mathrm{mg}$ ), through carbodiimide crosslinking chemistry by stirring with 1-Ethyl-3-(3-dimethylaminopropyl) carbodiimide (EDC, $2.9 \mathrm{mg}$ ) and Sulfo- $N$-hydroxysuccinimide (Sulfo-NHS, $8.22 \mathrm{mg}$ ) in Phosphate Buffered Saline (PBS, pH 7.4, $1.3 \mathrm{ml})$. Acrylamide $(689.5 \mathrm{mg}$ ) was added into the mixture and stirred for 2 hours in room temperature. Then, the crosslinker, 3(Acryloyloxy)-2-hydroxypropyl methacrylate (AHM, $321.3 \mathrm{mg}$ ) was added and vortexed to complete the monomer solution.

Separately, an oil phase was prepared, consisting of dioctyl sulfosuccinate sodium salt (1.6g) and Brij L4 (3.3 ml) dissolved in 45mL of hexanes. The full monomer solution was then injected and emulsified into this oil phase. To remove dissolved oxygen from the oil phase, it is purged with nitrogen for at least 20 minutes. Following the purge, polymerization was initiated by the addition of $\mathrm{N}, \mathrm{N}, \mathrm{N}^{\prime}, \mathrm{N}^{\prime}$-tetramethylethylenediamine (TEMED) $(100 \mu \mathrm{l})$ and $10 \%(\mathrm{w} / \mathrm{w})$ Ammonium Persulfate $(100 \mu \mathrm{l})$. The reaction was allowed to stir for 2 hours, after which hexane was removed by rotary evaporation. The prepared nanoparticles were washed with ethanol and water with Amicon Filter Cell using a $300 \mathrm{kDa}$ filter, and then lyophilized. Although slight leaching of the dye was observed initially, during purification, there was no leaching during further steps of the synthesis. The surface of the G2-PAA NP was PEGylated and conjugated with tumor homing F3 peptides, following the previous protocol. ${ }^{35}$ The cancer targeting capability of our PAAm NPs conjugated with F3 peptides has been extensively studied. ${ }^{31,35-38}$ Bi-functional Polyethylene Glycol (MAL-PEG-SCM, $2 \mathrm{kDa}$, Creative PEGWorks) (4 mg) was added into G2-PAA NP in PBS (pH 7.4) (50 mg per $2.5 \mathrm{ml})$. After 30 min of stirring, it was washed with PBS using Amicon Ultra Centrifugal Filter (100 kDa) and F3 Peptide (KDEPQRRSARLSAKPAPPKPEPKPKKAPAKKC, RS Synthesis) (11 mg) was added and stirred overnight. Cysteine $(0.63 \mathrm{mg})$ was added and stirred for 2 hours to deactivate unreacted maleimide groups. The NP solution was washed with water and lyophilized. The G2-PAA NP was characterized by UV-VIS spectroscopy (UV-1601 Spectrometer, Shimadzu), fluorescence spectroscopy (FluoroMax-3, Horiba), and Dynamic Light Scattering instrument (DLS, Delsa Nano C particle analyzer instrument, Beckman Coulter). The hydrodynamic size of G2-PAA NP was $\sim 60 \mathrm{~nm}$ in Figure S1.

\section{In vitro PALT calibration setup}

G2-PAA NP (5mg/ml in PBS) solution was stored in a vial and continuously flowed into an optically clear test tube (polyvinyl chloride, $1.58 \mathrm{~mm}$ I.D. and $3.17 \mathrm{~mm}$ O.D.) using a peristaltic pump sealed with rubber septum. A needle type oximeter (Microx TX3, Presens, Regensburg, Germany) was inserted directly into the NP solution and used to monitor the oxygen concentration for calibration. The oxygen concentration in each solution was controlled by bubbling the solution using nitrogen/air mixtures with different oxygen contents.

An optical parametric oscillator (OPO) pumped by the second harmonic of a pulsed neodymium-doped aluminum garnet (Nd:YAG) laser (Surelite OPO plus, Continuum, Santa Clara, CA) provided the pump beam at $630 \mathrm{~nm}$. Another Nd:YAG laser pumped OPO 
(Vibrant B, Opotek, Carlsbad, CA) provided the probe beam at $920 \mathrm{~nm}$. The two laser beams were overlapped onto the tube, while the triggers of the two lasers were controlled by a delay generator (DG535, Stanford Research Systems). PA signals corresponding to the pump pulse, the probe pulse, and the pump plus the probe pulses were collected using a cylindrically focused US transducer (V312, Panametrics, Waltham, MA). To extract the PA lifetime signal from the triplet state absorption of the G2 dye, the PA signal from the pump pulse and the PA signal from the probe pulse were subtracted from the PA signal from the pump plus the probe pulses, following the protocol described in our previous study. ${ }^{23}$ This step was repeated for different delay times $(10,20,30,40$, and $50 \mu \mathrm{s})$ between the pump pulse and the probe pulse. Then the extracted PA signals from the triplet state absorption correspondent to different delay times were fitted with an exponential decay curve. By doing this, the decay rate of the G2 dye in the PAAm NPs was quantified for the measurement of the ambient oxygen concentration.

\section{PALT in vivo imaging setup}

The setup for the lasers was similar to that for the in vitro PALT calibration. The laser beams covered the entire imaging region with the light fluence on the skin within the ANSI safety limit. PA imaging in vivo was performed using our US and PA dual imaging system built on a commercially available research US platform (V1, Verasonics, Kirkland, WA) and a linear array probe working at a central frequency of 11.25 MHz (CL15-7, Philips, Andover, MA). The details of this imaging system have been introduced in our former publication. ${ }^{39}$ The system can acquire 2D B-scan PA and US images of the same image plane simultaneously, both in truly real-time fashion at a frame rate of $10 \mathrm{~Hz}$. With the pump beam at 630-nm wavelength and the probe beam at $920-\mathrm{nm}$ wavelength, PA images, after 50 times averaging, were obtained at the different delay times so as to process the PALT oxygen images. In a final PA and US combined image, the B-scan US image shows the structural information of the in vivo sample in gray scale, while the PALT image demonstrating the oxygen concentrations in pseudo color scale is superimposed on the US image.

\section{PALT in vivo imaging procedure}

All the procedures on live animals were approved by the University Committee on the Use and Care of Animals (UCUCA) of the University of Michigan. Four nude mice (5 weeks old female, Athymic nude Fox/NU, Envigo) were used as controls for comparison with the results from tumor imaging. For each control mouse, the G2-PAA NP (20 mg/ml in PBS, 0.1 $\mathrm{ml})$ solution was injected locally into the thigh muscle of the mouse. The mouse was anesthetized with inhalation of 1.0-2.0\% isoflurane, mixing with pure oxygen (i.e. 100\%), or oxygen/nitrogen mixtures with $20 \%$ or $10 \%$ oxygen concentration. Twenty minutes after inhalation of each breathing gas, PALT images were taken.

To extract the PA lifetime signals from the triplet state absorption of the G2-PAA NPs, the PA image induced by the pump pulse and the PA image induced by the probe pulse were extracted from the PA image induced by the pump plus the probe pulses. This image subtraction was done pixel by pixel. This step was repeated for different delay times $(10,20$, 30,40 , and $50 \mu \mathrm{s}$ ) between the pump pulse and the probe pulse. For each pixel, the exponential decay curve was obtained from the extracted PA signals. The five signal 
intensities from the different delay times were fitted with an exponential decay curve. Then the decay rate of the exponential curve was used to quantify the ambient oxygen concentration at each image pixel.

Four nude mice ( 5 weeks old female, Athymic nude Fox/NU, Envigo) were used for tumor oxygen imaging. The 9L rat glioma cell line (American Type Culture Collection) was cultured in RPMI 1640 medium supplemented with 10\% Fetal Bovine Serum (FBS) and 1\% Antibiotic-antimycotic. Approximately $10^{6}$ cells in $100 \mu \mathrm{l}$ of culture media were subcutaneously injected on the back of each mouse. Each tumor was allowed to grow for 2-4 weeks until its volume reached around $0.5 \mathrm{cc}$, as measured using a caliper. The nude mice were anesthetized with the inhalation of 1.0-2.0\% isoflurane, mixing with pure oxygen. The PALT images were acquired $60 \mathrm{~min}$ after the tumor targeted G2-PAA NPs $(20 \mathrm{mg} / \mathrm{ml}$ in PBS, $250 \mathrm{mg}$ NP per kg body weight) were injected systemically via the tail vein. PALT images were obtained using the same method described in the above. In addition, PA images at $576 \mathrm{~nm}, 584 \mathrm{~nm}$, and $630 \mathrm{~nm}$ were also taken in order to derive the images showing the spatially distributed G2-PAA NPs in the imaging area. The images at the three wavelengths was used to decouple the three major absorbers in this application, including G2-PAA NPs, the oxygenated hemoglobin, and the deoxygenated hemoglobin, by conducting multispectral PA imaging, as described in previous publications. ${ }^{26,40,41}$

\section{Supplementary Material}

Refer to Web version on PubMed Central for supplementary material.

\section{ACKNOWLEDGMENT}

This work was supported by NIH/NCI grant R01CA186769.

\section{REFERENCES}

1. Petrova V; Annicchiarico-Petruzzelli M; Melino G; Amelio I, The Hypoxic Tumour Microenvironment. Oncogenesis 2018, 7, 10. [PubMed: 29362402]

2. Brown JM, Tumor Hypoxia in Cancer Therapy. Methods Enzymol. 2007, 435, 297-321. [PubMed: 17998060]

3. Zhong H; De Marzo AM; Laughner E; Lim M; Hilton DA; Zagzag D; Buechler P; Isaacs WB; Semenza GL; Simons JW, Overexpression of Hypoxia-Inducible Factor 1a in Common Human Cancers and Their Metastases. Cancer Res. 1999, 59, 5830-5835. [PubMed: 10582706]

4. Maxwell PH; Wiesener MS; Chang GW; Clifford SC; Vaux EC; Cockman ME; Wykoff CC; Pugh CW; Maher ER; Ratcliffe PJ, The Tumour Suppressor Protein VHL Targets Hypoxia-Inducible Factors for Oxygen-Dependent Proteolysis. Nature (London, U. K.) 1999, 399, 271-275. [PubMed: 10353251]

5. Dewhirst MW; Birer SR, Oxygen-Enhanced MRI Is a Major Advance in Tumor Hypoxia Imaging. Cancer Res. 2016, 76, 769-772. [PubMed: 26837768]

6. Frackowiak RS; Lenzi GL; Jones T; Heather JD, Quantitative Measurement of Regional Cerebral Blood Flow and Oxygen Metabolism in Man Using 150 and Positron Emission Tomography: Theory, Procedure, and Normal Values. Journal of computer assisted tomography 1980, 4, 727-736. [PubMed: 6971299]

7. Wang X-d.; Wolfbeis, O. S., Optical Methods for Sensing and Imaging Oxygen: Materials, Spectroscopies and Applications. Chem. Soc. Rev 2014, 43, 3666-3761. [PubMed: 24638858] 
8. Jo J; Lee CH; Kopelman R; Wang X, In Vivo Quantitative Imaging of Tumor pH by Nanosonophore Assisted Multispectral Photoacoustic Imaging. Nat. Commun 2017, 8, 471. [PubMed: 28883396]

9. Lee CH; Folz J; Zhang W; Jo J; Tan JWY; Wang X; Kopelman R, Ion-Selective Nanosensor for Photoacoustic and Fluorescence Imaging of Potassium. Anal. Chem 2017, 89, 7943-7949. [PubMed: 28633520]

10. Lee CH; Folz J; Zhang W; Jo J; Tan JWY; Wang X; Kopelman R, Correction to Ion-Selective Nanosensor for Photoacoustic and Fluorescence Imaging of Potassium. Anal. Chem 2017, 89, 13674. [PubMed: 29185713]

11. Ashkenazi S; Huang SW; Horvath T; Koo YE; Kopelman R, Photoacoustic Probing of Fluorophore Excited State Lifetime with Application to Oxygen Sensing. J. Biomed. Opt 2008, 13, 034023. [PubMed: 18601568]

12. Horvath TD; Kim G; Kopelman R; Ashkenazi S, Ratiometric Photoacoustic Sensing of pH Using A "Sonophore". Analyst 2008, 133, 747-749. [PubMed: 18493674]

13. Shao Q; Ashkenazi S, Photoacoustic Lifetime Imaging for Direct In Vivo Tissue Oxygen Monitoring. J. Biomed. Opt 2015, 20, 036004. [PubMed: 25748857]

14. Shao Q; Morgounova E; Jiang C; Choi J; Bischof J; Ashkenazi S, In Vivo Photoacoustic Lifetime Imaging of Tumor Hypoxia in Small Animals. J. Biomed. Opt 2013, 18, 076019. [PubMed: 23877772]

15. Ray A; Rajian JR; Lee Y-EK; Wang X; Kopelman R, Lifetime-Based Photoacoustic Oxygen Sensing In Vivo. J. Biomed. Opt 2012, 17, 057004. [PubMed: 22612143]

16. Cash KJ; Li C; Xia J; Wang LV; Clark HA, Optical Drug Monitoring: Photoacoustic Imaging of Nanosensors to Monitor Therapeutic Lithium In Vivo. ACS Nano 2015, 9, 1692-1698. [PubMed: 25588028]

17. Chen Q; Liu X; Chen J; Zeng J; Cheng Z; Liu Z, A Self-Assembled Albumin-Based Nanoprobe for In Vivo Ratiometric Photoacoustic pH Imaging. Adv. Mater. (Weinheim, Ger.) 2015, 27, 6820 6827.

18. Morales J; Pawle RH; Akkilic N; Luo Y; Xavierselvan M; Albokhari R; Calderon IAC; Selfridge S; Minns R; Takiff L; Mallidi S; Clark HA, DNA-Based Photoacoustic Nanosensor for Interferon Gamma Detection. ACS Sens. 2019, 4, 1313-1322. [PubMed: 30973005]

19. Wang X; Pang Y; Ku G; Xie X; Stoica G; Wang LV, Noninvasive Laser-Induced Photoacoustic Tomography for Structural and Functional In Vivo Imaging of The Brain. Nat. Biotechnol 2003, 21, 803-806. [PubMed: 12808463]

20. Tzoumas S; Nunes A; Olefir I; Stangl S; Symvoulidis P; Glasl S; Bayer C; Multhoff G; Ntziachristos V, Eigenspectra Optoacoustic Tomography Achieves Quantitative Blood Oxygenation Imaging Deep in Tissues. Nat. Commun 2016, 7, 12121. [PubMed: 27358000]

21. Yao J; Wang L; Yang J-M; Maslov KI; Wong TTW; Li L; Huang C-H; Zou J; Wang LV, HighSpeed Label-Free Functional Photoacoustic Microscopy of Mouse Brain in Action. Nat. Methods 2015, 12, 407. [PubMed: 25822799]

22. Dunphy I; Vinogradov SA; Wilson DF, Oxyphor R2 and G2: Phosphors for Measuring Oxygen by Oxygen-Dependent Quenching of Phosphorescence. Anal. Biochem 2002, 310, 191-198. [PubMed: 12423638]

23. Tan JWY; Lee CH; Kopelman R; Wang X, Transient Triplet Differential (TTD) Method for Background Free Photoacoustic Imaging. Sci. Rep 2018, 8, 9290. [PubMed: 29915177]

24. Ashkenazi S, Photoacoustic Lifetime Imaging of Dissolved Oxygen Using Methylene Blue. J. Biomed. Opt 2010, 15, 040501. [PubMed: 20799768]

25. Gonzalez-Bejar M; Montes-Navajas P; Garcia H; Scaiano JC, Methylene Blue Encapsulation in Cucurbit[7]uril: Laser Flash Photolysis and Near-IR Luminescence Studies of The Interaction with Oxygen. Langmuir 2009, 25, 10490-10494. [PubMed: 19735127]

26. Lemaster JE; Jokerst JV, What Is New in Nanoparticle-Based Photoacoustic Imaging? Wiley Interdiscip. Rev.: Nanomed. Nanobiotechnol 2017, 9, e1404.

27. Esipova TV; Karagodov A; Miller J; Wilson DF; Busch TM; Vinogradov SA, Two New "Protected" Oxyphors for Biological Oximetry: Properties and Application in Tumor Imaging. Anal. Chem 2011, 83, 8756-8765. [PubMed: 21961699] 
28. Lee Y-EK; Ulbrich EE; Kim G; Hah H; Strollo C; Fan W; Gurjar R; Koo S; Kopelman R, Near Infrared Luminescent Oxygen Nanosensors with Nanoparticle Matrix Tailored Sensitivity. Anal. Chem 2010, 82, 8446-8455. [PubMed: 20849084]

29. Lee CH; Jo J; Wang X; Kopelman R, CHAPTER 10 Photoacoustic Imaging of Oxygen. In Quenched-phosphorescence Detection of Molecular Oxygen: Applications in Life Sciences, The Royal Society of Chemistry: 2018; pp 205-219.

30. Harris JM; Chess RB, Effect of Pegylation on Pharmaceuticals. Nat. Rev. Drug Discovery 2003, 2, 214-221. [PubMed: 12612647]

31. Reddy GR; Bhojani MS; McConville P; Moody J; Moffat BA; Hall DE; Kim G; Koo YE; Woolliscroft MJ; Sugai JV; Johnson TD; Philbert MA; Kopelman R; Rehemtulla A; Ross BD, Vascular Targeted Nanoparticles for Imaging and Treatment of Brain Tumors. Clin. Cancer Res. 2006, 12, 6677-6686. [PubMed: 17121886]

32. Lee CH; Folz J; Tan JWY; Jo J; Wang X; Kopelman R, Chemical Imaging In Vivo: PhotoacousticBased 4-Dimensional Chemical Analysis. Anal. Chem 2019, 91, 2561-2569. [PubMed: 30638359]

33. Li M; Tang Y; Yao J, Photoacoustic Tomography of Blood Oxygenation: A Mini Review. Photoacoustics 2018, 10, 65-73. [PubMed: 29988848]

34. Samuel J; Franklin C, Hypoxemia and Hypoxia In Common Surgical Diseases: An Algorithmic Approach to Problem Solving, Myers JA; Millikan KW; Saclarides TJ, Eds. Springer New York: New York, NY, 2008; pp 391-394.

35. Nie G; Hah HJ; Kim G; Lee Y-EK; Qin M; Ratani TS; Fotiadis P; Miller A; Kochi A; Gao D; Chen T; Orringer DA; Sagher O; Philbert MA; Kopelman R, Hydrogel Nanoparticles with Covalently Linked Coomassie Blue for Brain Tumor Delineation Visible to The Surgeon. Small 2012, 8, 884891. [PubMed: 22232034]

36. Qin M; Hah HJ; Kim G; Nie G; Lee Y-EK; Kopelman R, Methylene Blue Covalently Loaded Polyacrylamide Nanoparticles for Enhanced Tumor-Targeted Photodynamic Therapy. Photochem. Photobiol. Sci 2011, 10, 832-841. [PubMed: 21479315]

37. Ray A; Wang X; Lee Y-E; Hah H; Kim G; Chen T; Orringer D; Sagher O; Liu X; Kopelman R, Targeted Blue Nanoparticles as Photoacoustic Contrast Agent for Brain Tumor Delineation. Nano Res. 2011, 4, 1163-1173. [PubMed: 29423130]

38. Karamchand L; Kim G; Wang S; Hah HJ; Ray A; Jiddou R; Koo Lee Y-E; Philbert MA; Kopelman R, Modulation of Hydrogel Nanoparticle Intracellular Trafficking by Multivalent Surface Engineering with Tumor Targeting Peptide. Nanoscale 2013, 5, 10327-10344. [PubMed: 24056573]

39. Yuan J; Xu G; Yu Y; Zhou Y; Carson PL; Wang X; Liu X, Real-Time Photoacoustic and Ultrasound Dual-Modality Imaging System Facilitated with Graphics Processing Unit and Code Parallel Optimization. J. Biomed. Opt 2013, 18, 86001. [PubMed: 23907277]

40. Wang XD; Xie XY; Ku GN; Wang LHV, Noninvasive Imaging of Hemoglobin Concentration and Oxygenation in The Rat Brain Using High-Resolution Photoacoustic Tomography. J. Biomed. Opt 2006, 11, 024015. [PubMed: 16674205]

41. Cox B; Laufer JG; Arridge SR; Beard PC, Quantitative Spectroscopic Photoacoustic Imaging: A Review. J. Biomed. Opt 2012, 17, 061202. [PubMed: 22734732] 
(a)

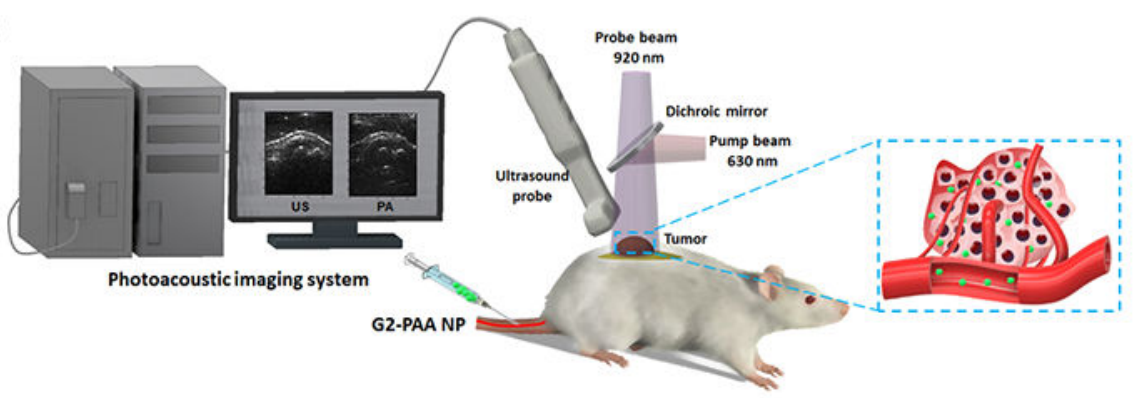

(b)

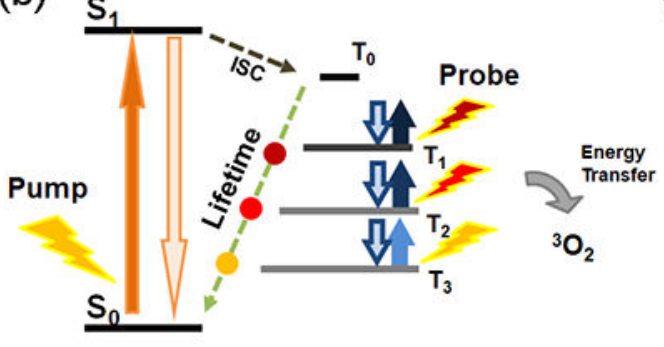

(c)

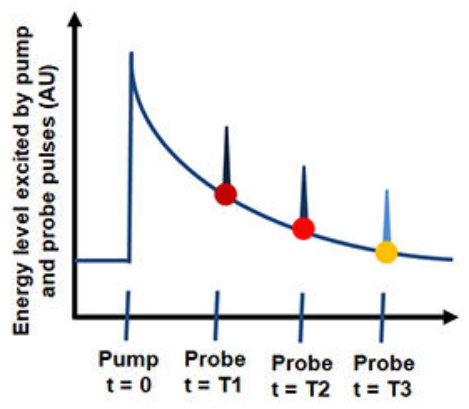

Figure 1.

PALT based oxygen imaging schematic. (a) Illustration of PALT based oxygen imaging of a tumor in vivo. (b) The pump pulse excites the tumor targeted G2-PAA NPs and the probe pulse excites these nanoparticles decaying to ground level at different delay times. (c) By changing the delay time between the pump and the probe pulses, the exponential decay rate of the phosphorescence, which is linearly correlated with the ambient oxygen concentration, can be measured. 
(a)
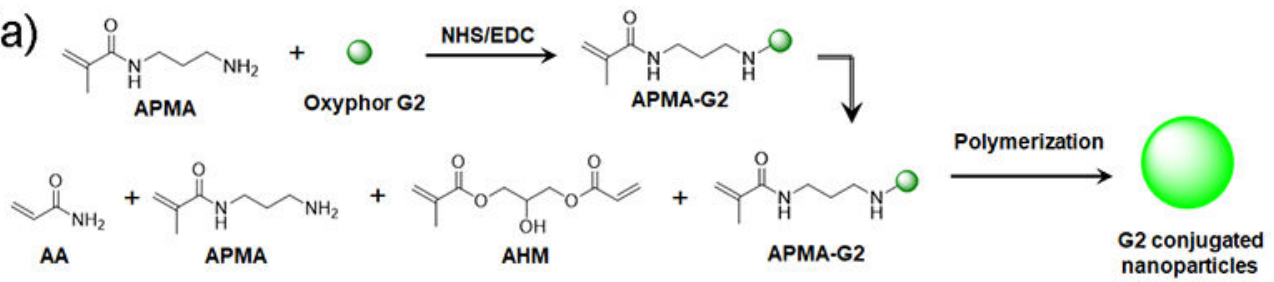

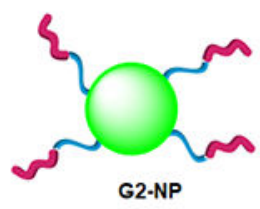

(b)

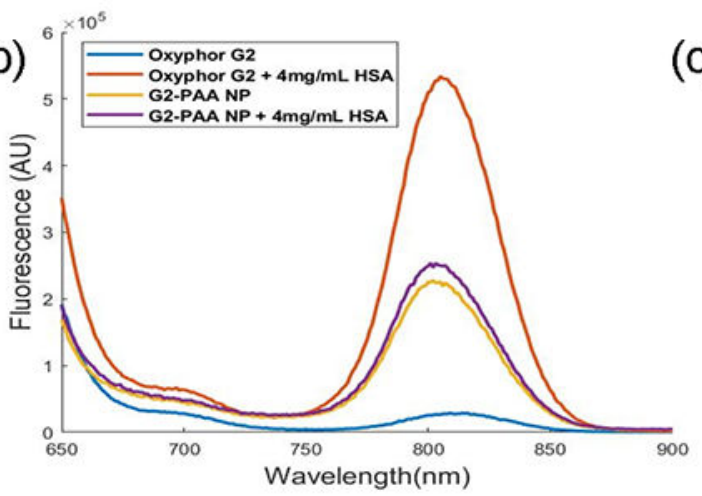

Tumor Homing - F3 peptide (KDEPQRRSARLSAKPAPPKPEPKPKKAPAKKC)
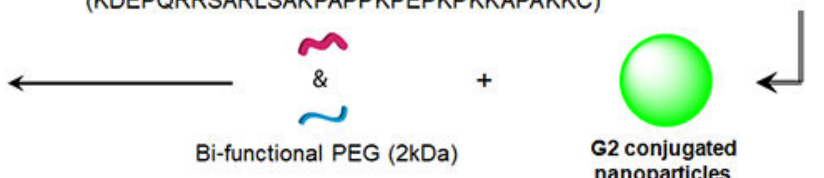

(c)

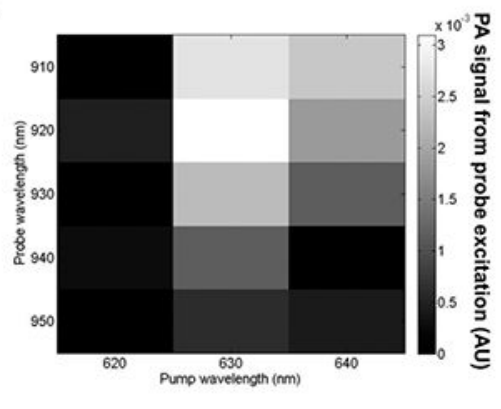

Figure 2.

(a) G2-PAA NP synthetic scheme. (b) Fluorescence emission of oxyphor G2 free dye and G2-PAA NPs with and without the presence of human serum albumin (HSA). (c) PA lifetime signal amplitude as a function of the pump beam wavelength and the probe beam wavelength. 

(a) $02 \mathrm{mg} / \mathrm{ml}$
(b) $05 \mathrm{mg} / \mathrm{ml}$
(C) $20 \mathrm{mg} / \mathrm{ml}$
(d) $05 \mathrm{mg} / \mathrm{ml}+\mathrm{HSA}$
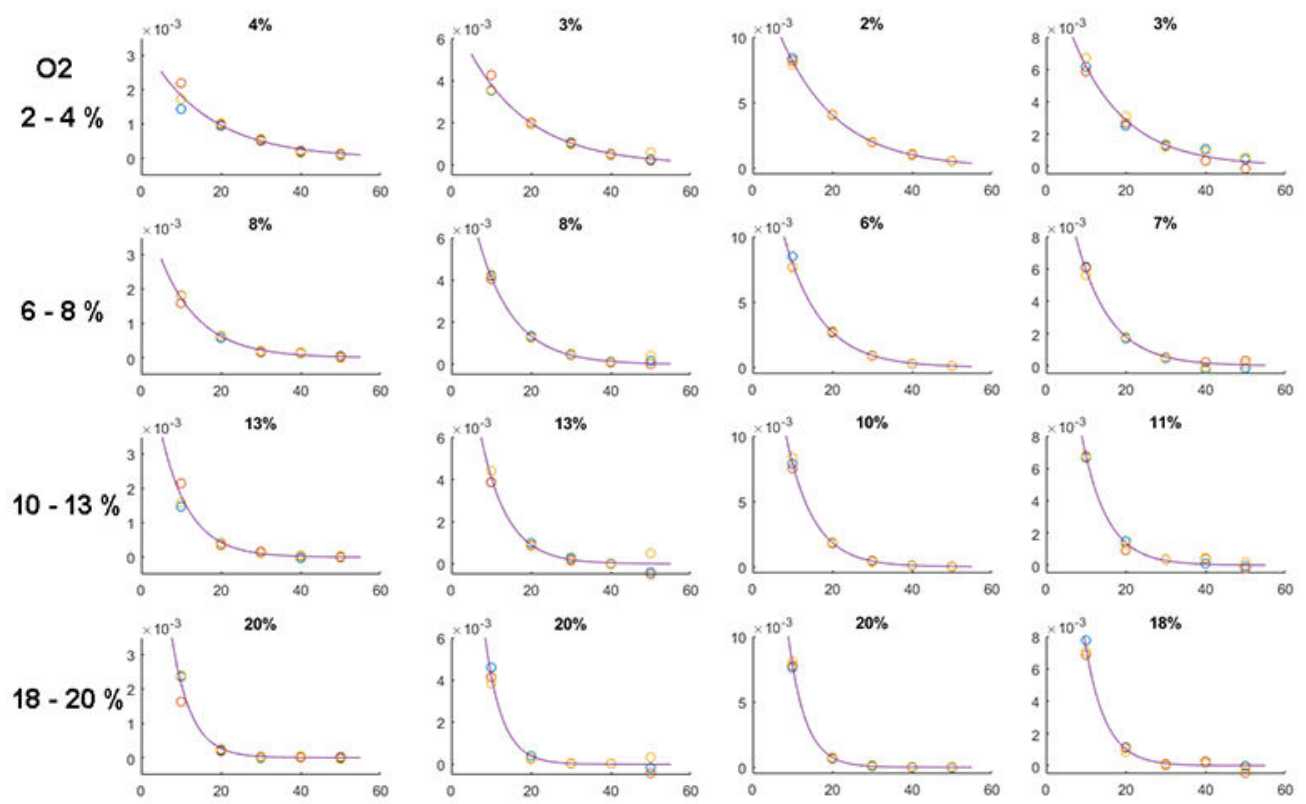

Figure 3.

PA measurement of the triplet state absorption of G2-PAA NPs as a function of the delay time between the pump and the probe pulses. Each panel presents the result for different ambient oxygen concentration (grouped into four ranges) and different G2-PAA NP concentrations as well as with the presence of HSA. (a)-(c) The concentrations of G2-PAA NPs in PBS pH 7.4 are 2, 5, and $20 \mathrm{mg} / \mathrm{ml}$, respectively. (d) $5 \mathrm{mg} / \mathrm{ml} \mathrm{G2-PAA} \mathrm{NP} \mathrm{mixed}$ with HSA $(4 \mathrm{mg} / \mathrm{ml})$. 

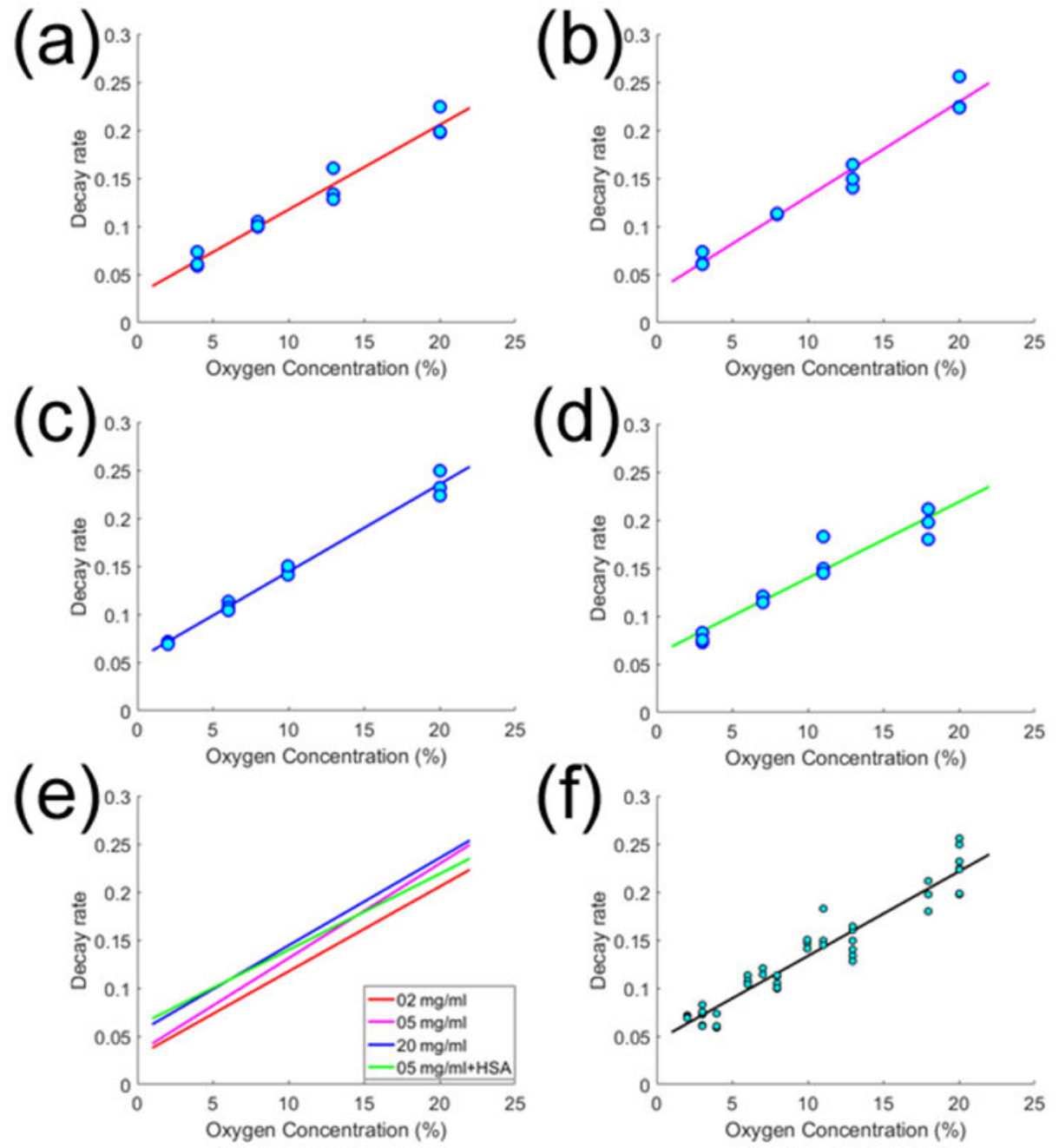

Figure 4.

Quantified decay rates from the PA measurement of the triplet state absorption of G2-PAA NPs as a function of the ambient oxygen concentration. (a)-(d) are the results for different G2-PAA NP concentrations as well as with the presence of HSA. In each panel, a linear fit is made, showing by the solid line. The linear fitting lines from (a)-(d) are compared in (e). (f) With all the PA measurements in (a)-(d), a linear fit is made. 

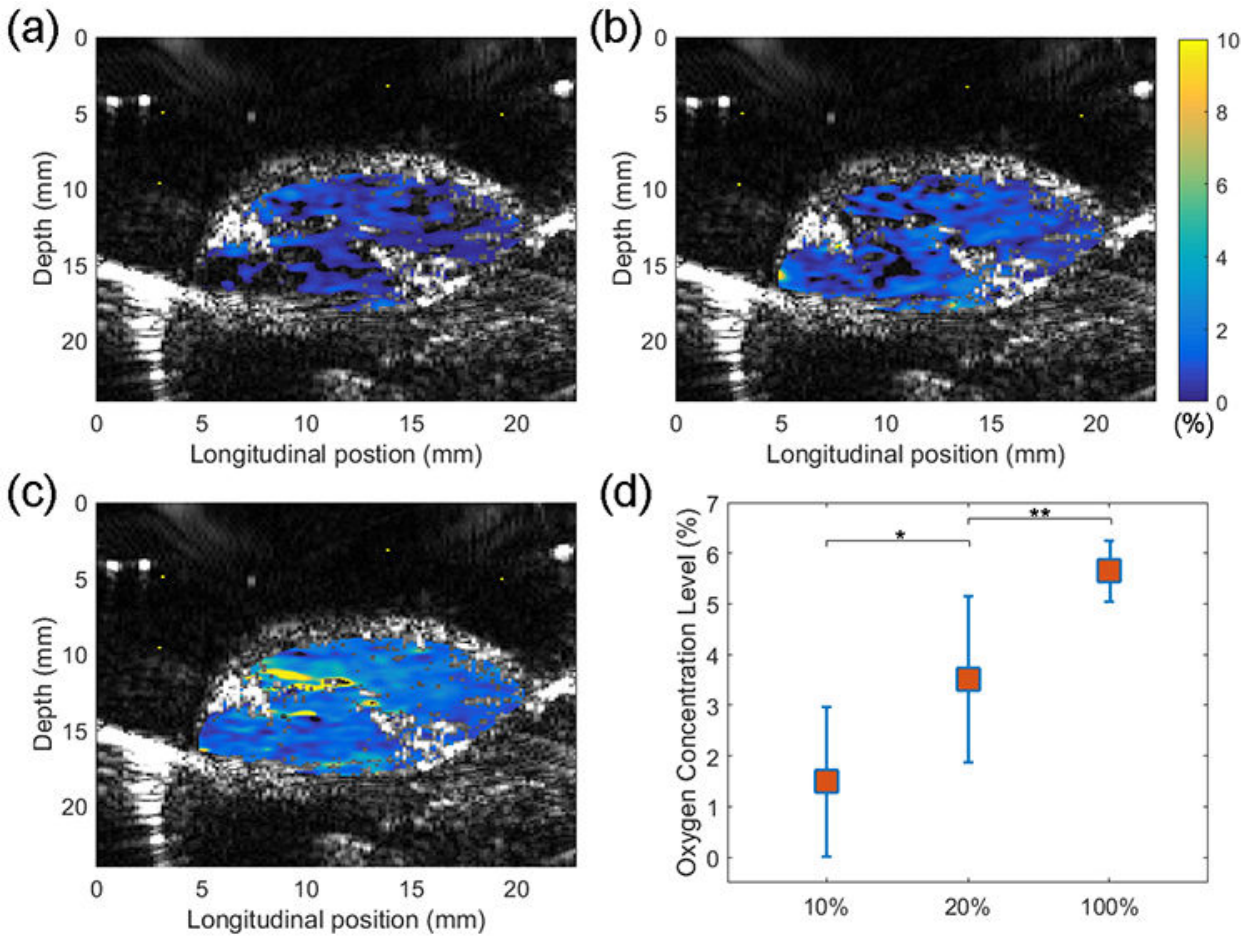

Figure 5.

PALT based oxygen imaging of thigh muscle in vivo. Oxygen concentration images in (a)(c) were acquired when oxygen contents in the breathing gas were 10\%, 20\%, and 100\%, respectively. In each panel, the PALT image demonstrating the oxygen concentrations in pseudo color scale is superimposed on the US image showing the tissue structure. (d) Average oxygen concentrations in the thigh muscles for $10 \%, 20 \%$ and $100 \%$ oxygen contents in the breathing gas $(\mathrm{n}=4)$ with standard deviations. $*$ and $* *$ are for $\mathrm{p}<0.05$. 
(a) 0

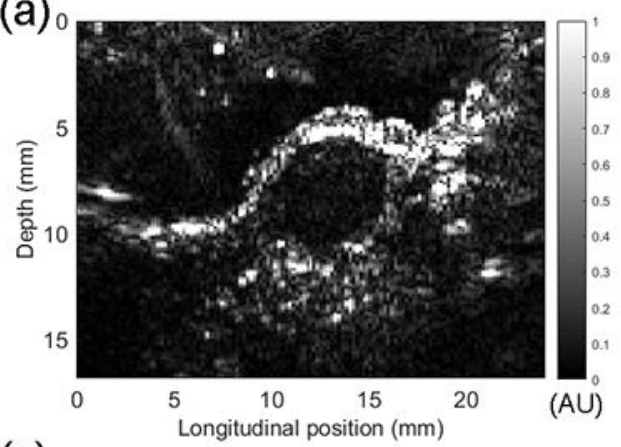

(c) 0

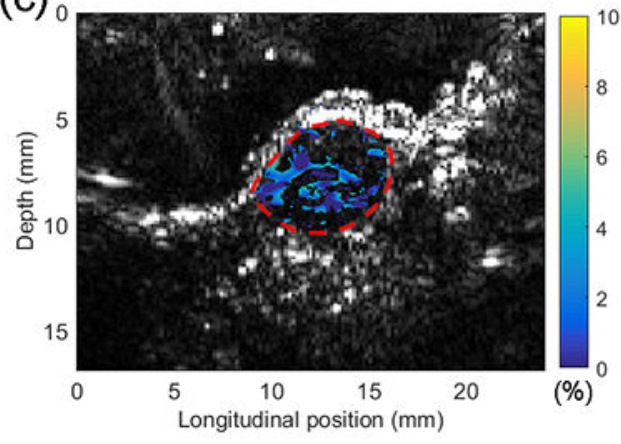

(b)

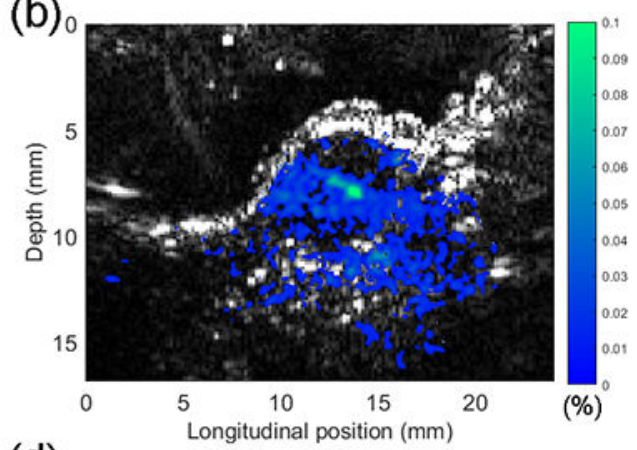

(d)

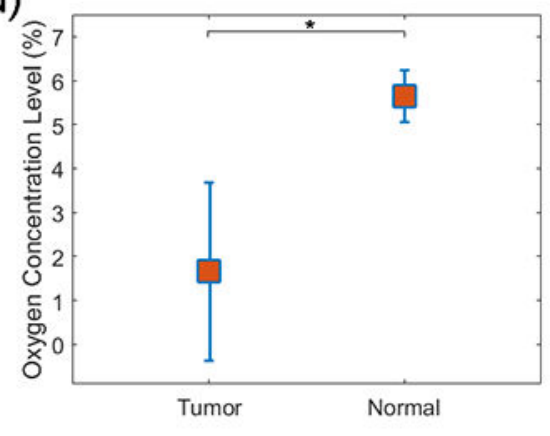

Figure 6.

Imaging results from a mouse tumor in vivo. (a) Ultrasound image of the tumor. (b) PA image showing the distribution of G2-PAA NPs in the imaging area including the tumor. The spatially distributed NP concentration was derived from multispectral PA imaging at 576 $\mathrm{nm}, 584 \mathrm{~nm}$, and $630 \mathrm{~nm}$ wavelengths. (c) PALT oxygen image in the tumor area. (d) Average oxygen concentrations in the tumors $(n=4)$ vs. average oxygen concentration in the thigh muscles $(n=4)$ with standard deviations, both measured with $100 \%$ oxygen content in the breathing gas. $*$ is for $\mathrm{p}<0.01$. 
(a)

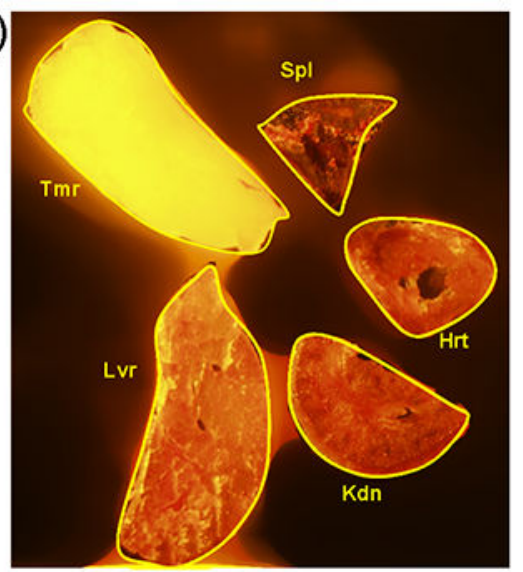

(b)

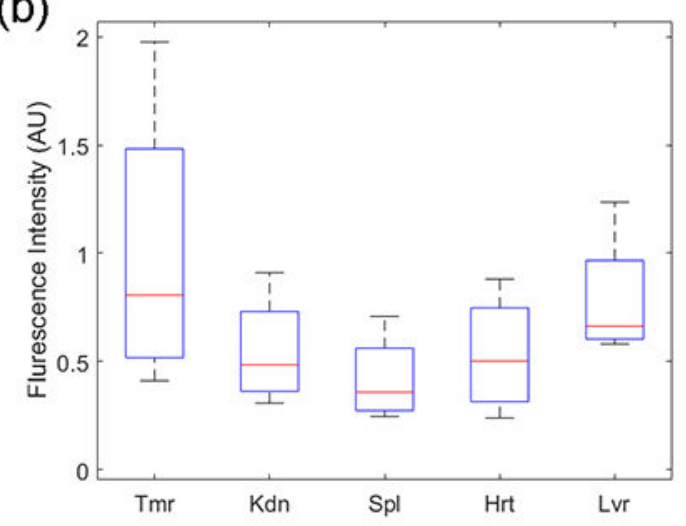

Figure 7.

Organ accumulations of G2-PAA NPs after systemic injection via the tail vein. (a) A fluorescence image of dissected organs $(\mathrm{Tmr}=$ tumor, $\mathrm{Kdn}=$ kidney, $\mathrm{Spl}=$ spleen, $\mathrm{Hrt}=$ heart, and Lvr $=$ liver). (b) Boxplot showing the statistical distributions of average fluorescence intensities of the dissected organs. 